\title{
BMJ open Grey and white matter abnormalities in chronic obstructive pulmonary disease: a case-control study
}

\author{
Haiyan Zhang, ${ }^{1}$ Xiaochuan Wang, ${ }^{2}$ Jianzhong Lin, ${ }^{3}$ Yinchuan Sun, ${ }^{1}$ \\ Yongxia Huang, ${ }^{1}$ Tianhe Yang, ${ }^{3}$ Shili Zheng, ${ }^{4}$ Ming Fan, ${ }^{5}$ Jiaxing Zhang ${ }^{1}$
}

To cite: Zhang $\mathrm{H}$, Wang $\mathrm{X}$, Lin J, et al. Grey and white matter abnormalities in chronic obstructive pulmonary disease: a case-control study. BMJ Open 2012;2:e000844. doi:10.1136/

bmjopen-2012-000844

- Prepublication history for this paper is available online. To view these files please visit the journal online (http:// dx.doi.org/10.1136/ bmjopen-2012-000844).

$\mathrm{HZ}$ and $\mathrm{XW}$ contributed equally to this work.

Received 12 January 2012 Accepted 6 March 2012

This final article is available for use under the terms of the Creative Commons Attribution Non-Commercial 2.0 Licence; see http://bmjopen.bmj.com

For numbered affiliations see end of article.

Correspondence to Dr Jiaxing Zhang; zhangjiaxing@xmu.edu.cn

\section{ABSTRACT}

Objectives: The irreversible airflow limitation characterised by chronic obstructive pulmonary disease (COPD) causes a decrease in the oxygen supply to the brain. The aim of the present study was to investigate brain structural damage in COPD.

Design: Retrospective case-control study. Patients with COPD and healthy volunteers were recruited. The two groups were matched in age, gender and educational background.

Setting: A hospital and a number of communities: they are all located in southern Fujian province, China.

Participants: 25 stable patients and 25 controls were enrolled from December 2009 to May 2011.

Methods: Using voxel-based morphometry and tractbased spatial statistics based on MRI to analyse grey matter (GM) density and white matter fractional anisotropy (FA), respectively, and a battery of neuropsychological tests were performed.

Results: Patients with COPD (vs controls) showed decreased GM density in the limbic and paralimbic structures, including right gyrus rectus, left precentral gyrus, bilateral anterior and middle cingulate gyri, bilateral superior temporal gyri, bilateral anterior insula extending to Rolandic operculum, bilateral thalamus/ pulvinars and left caudate nucleus. Patients with COPD (vs controls) had decreased FA values in the bilateral superior corona radiata, bilateral superior and inferior longitudinal fasciculus, bilateral optic radiation, bilateral lingual gyri, left parahippocampal gyrus and fornix. Lower FA values in these regions were associated with increased radial diffusivity and no changes of longitudinal diffusivity. Patients with COPD had poor performances in the Mini-Mental State Examination, figure memory and visual reproduction. GM density in some decreased regions in COPD had positive correlations with arterial blood $\mathrm{PO}_{2}$, negative correlations with disease duration and also positive correlations with visual tasks.

Conclusion: The authors demonstrated that COPD exhibited loss of regional GM accompanied by impairment of white matter microstructural integrity, which was associated with disease severity and may underlie the pathophysiological and psychological changes of COPD.

\section{ARTICLE SUMMARY}

Article focus

- Decreased oxygen supply to brain may cause neuronal damage in COPD. However, the damage remains largely uninvestigated.

Key messages

- We found that COPD extends to the brain, with the loss of regional cortical grey matter accompanied by impairment in the white matter microstructural integrity.

- Our findings would be help for clinical therapy of COPD.

Strengths and limitations of this study

- Multiple analyses were used based on MR images. The statistic power for FA analysis was weak.

\section{INTRODUCTION}

Chronic obstructive pulmonary disease (COPD) continues to be a major cause of morbidity and mortality. It is increasingly recognised that COPD extends beyond the lung. ${ }^{1}$ The irreversible airflow limitation characterised by COPD usually develops arterial oxygen desaturation, which could subsequently result in a decrease in oxygen transport to the brain. Hypoxia during COPD has been previously proven to induce cerebral perfusion decline ${ }^{2}$ and metabolic changes. ${ }^{3-6}$ Moreover, systematic inflammation $^{1}$ may also cause neuronal damages in the brain of patients with COPD. In patients with COPD, clinical symptoms such as neuropsychological deficits, ${ }^{7}$ depression and anxiety, ${ }^{1}$ and physical disability ${ }^{1}$ have been well documented. Taken together, these data suggest the presence of brain structural alteration. However, until now, it remains largely uninvestigated.

Voxel-based morphometry (VBM) and Tract-Based Spatial Statistics (TBSS) ${ }^{8}$ based on MRI were adopted to measure grey matter (GM) density and white matter (WM) fibrous microstructure properties in tracts, 
Table 1 Demographic characteristics of the patients with COPD and healthy volunteers

\begin{tabular}{|c|c|c|c|}
\hline & $\begin{array}{l}\text { Patients } \\
\text { with COPD }\end{array}$ & Controls & p Value \\
\hline Number of subjects & 25 & 25 & \\
\hline Gender (female), \% & 16 & 16 & \\
\hline $\begin{array}{l}\text { Age (years), } \\
\text { mean } \pm S D \text { (range) }\end{array}$ & $\begin{array}{l}69.2 \pm 8.1 \\
(58-84)\end{array}$ & $\begin{array}{l}67.96 \pm 8.0 \\
(57-86)\end{array}$ & 0.59 \\
\hline $\begin{array}{l}\text { Education (years), } \\
\text { mean } \pm S D\end{array}$ & $6.7 \pm 3.9$ & $7.5 \pm 5.0$ & 0.53 \\
\hline $\begin{array}{l}\text { Family history of } \\
\text { COPD, \% }\end{array}$ & 4 & - & \\
\hline $\begin{array}{l}\text { Disease duration } \\
\text { (years) }\end{array}$ & $7.0 \pm 5.7$ & - & \\
\hline Actual smokers, \% & 44 & 40 & 0.86 \\
\hline
\end{tabular}

respectively. VBM is an automatic quantitative volumetric technique over the whole brain using voxel by voxel analysis without prior specification of regions-of-interest for analysis, and it does not rely on arbitrarily predefined structures. Recently, the preprocessing steps of VBM have been improved with the Diffeomorphic Anatomical Registration Through Exponentiated Lie algebra (DARTEL) registration method, ${ }^{9}$ which can achieve more accurate intersubject registration of brain images.

Table 2 Physiological and psychological characteristics

\begin{tabular}{|c|c|c|c|}
\hline & $\begin{array}{l}\text { Patients with } \\
\text { COPD }\end{array}$ & Controls & p Value \\
\hline BMI $\left(\mathrm{kg} / \mathrm{m}^{2}\right)$ & $20.8 \pm 3.9$ & $22.6 \pm 2.6$ & 0.081 \\
\hline ADL & $20.1 \pm 6.5$ & $14.6 \pm 1.5$ & $<0.001$ \\
\hline Heart rate & $92.4 \pm 16.2$ & $70.9 \pm 8.9$ & $<0.001$ \\
\hline \multicolumn{4}{|c|}{ Blood pressure $(\mathrm{mm} \mathrm{Hg})$} \\
\hline Systolic pressure & $136.1 \pm 19.1$ & $136.5 \pm 18.0$ & 0.940 \\
\hline Diastolic pressure & $81.8 \pm 10.7$ & $77.5 \pm 14.5$ & 0.307 \\
\hline \multicolumn{4}{|c|}{ Haematological measurements } \\
\hline $\mathrm{SaO}_{2}, \%$ & $94.0 \pm 4.2$ & $97.0 \pm 1.3$ & 0.003 \\
\hline $\mathrm{PO}_{2}(\mathrm{~mm} \mathrm{Hg})$ & $79.9 \pm 23.3$ & $98.5 \pm 11.3$ & 0.006 \\
\hline $\mathrm{PcO}_{2}(\mathrm{~mm} \mathrm{Hg})$ & $48.1 \pm 6.0$ & $39.8 \pm 3.0$ & $<0.001$ \\
\hline $\mathrm{pH}$ & $7.37 \pm 0.1$ & $7.4 \pm 0.01$ & 0.731 \\
\hline \multicolumn{4}{|c|}{ Pulmonary function testing } \\
\hline $\begin{array}{l}\text { Respiratory rate } \\
\text { (breaths/min) }\end{array}$ & $23.5 \pm 6.0$ & $16.9 \pm 5.5$ & 0.003 \\
\hline FVC (\% predicted) & $66.6 \pm 17.2$ & $96.1 \pm 14.7$ & $<0.001$ \\
\hline $\mathrm{FEV}_{1}(\%$ predicted $)$ & $43.4 \pm 16.4$ & $97.5 \pm 16.9$ & $<0.001$ \\
\hline $\mathrm{FEV}_{1} / \mathrm{FVC}, \%$ & $50.3 \pm 10.7$ & $80.0 \pm 8.3$ & $<0.001$ \\
\hline \multicolumn{4}{|l|}{ Cognitive tests } \\
\hline MMSE & $23.3 \pm 3.4$ & $25.8 \pm 2.1$ & 0.021 \\
\hline \multicolumn{4}{|l|}{ Digit span } \\
\hline Forward task & $7.0 \pm 1.6$ & $7.7 \pm 1.4$ & 0.125 \\
\hline Backward task & $4.1 \pm 1.9$ & $4.3 \pm 1.5$ & 0.612 \\
\hline Visual reproduction & $8.2 \pm 3.4$ & $10.3 \pm 3.0$ & 0.031 \\
\hline Figure memory & $10.5 \pm 3.0$ & $12.4 \pm 1.9$ & 0.010 \\
\hline
\end{tabular}

Data are presented as mean \pm SD.

$\mathrm{ADL}$, activities of daily living; BMI, body mass index; COPD,

chronic obstructive pulmonary disease; FVC, forced vital capacity $\mathrm{FEV}_{1}$, forced expired volume in one second; MMSE, Mini-Mental State Examination.

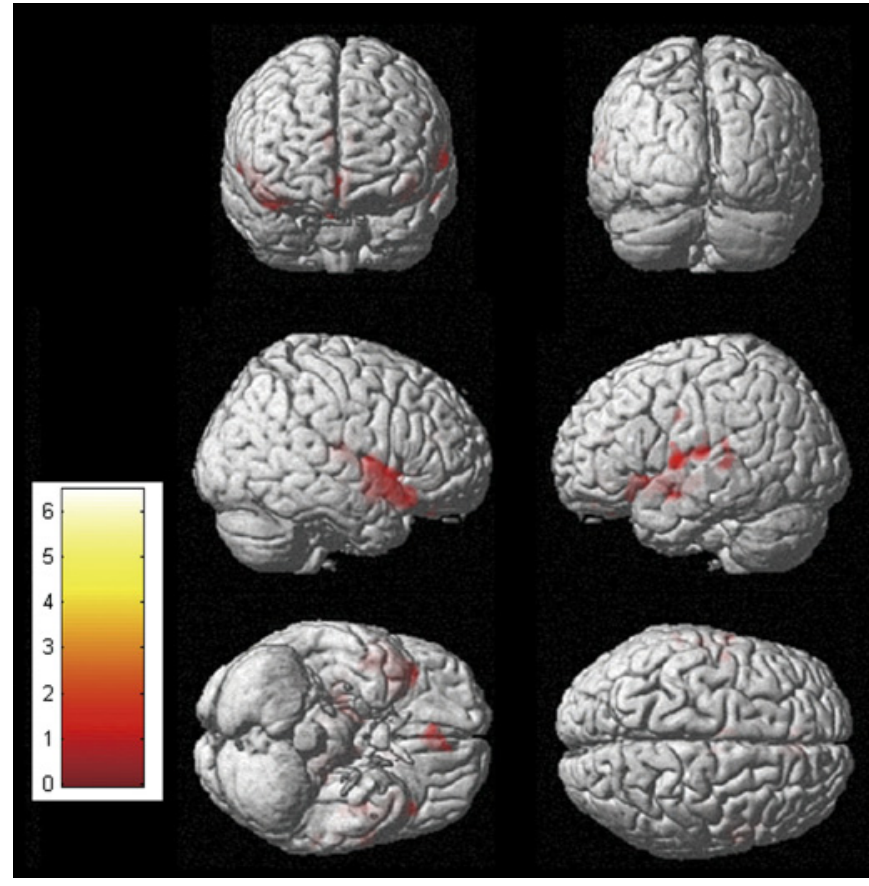

Figure 1 A statistical parametric map for grey matter density reduced in patients with chronic obstructive pulmonary disease versus healthy controls (pFDR_corrected $<0.01$ ) overlaid on the Montreal Neurological Institute template.

TBSS is a recently introduced method, which uses diffusion tensor MR imaging (DTI) to measure differences in fractional anisotropy (FA) between groups. TBSS increases the sensitivity and the interpretability of the results compared with voxel-based approaches based purely on non-linear registration. ${ }^{8}$ Moreover, diffusion tensor eigenvalues were also included in the analysis since they can help interpret FA changes in WM tracts by providing information regarding likely alterations in the proportion of longitudinally versus obliquely aligned myelinated fibres. The VBM and TBSS methods have been extensively applied in clinical researches, including the evaluation of morphological characteristics of high-altitude residents in our previous study. ${ }^{10}$

Dyspnoea is the most common complaint and most disabling symptom in patients with COPD. Functional MRI studies on breathlessness, air hunger and inspiratory loaded breathing have revealed that a large number of brain regions, including the frontal cortex, parietal cortex, temporal cortex, limbic cortex, cerebellar cortex and brainstem were activated by dyspnoea. ${ }^{11}$ These dyspnoea-activated brain regions have been shown to be impaired in patients with congenital central hypoventilation syndrome, ${ }^{12}$ in patients with obstructive sleep apnoea ${ }^{13}$ and in high-altitude residents. ${ }^{10}$ We therefore hypothesised that patients with COPD would have similar cerebral impairment.

\section{METHODS}

Subjects

Twenty-five patients were enrolled from December 2009 to May 2011. All patients had undergone a period of 
Figure 2 Grey matter density decrease in patients with chronic obstructive pulmonary disease versus healthy controls. Three-dimensional slices depicting regions showing reduced grey matter in the right gyrus rectus, left precentral gyrus, left Rolandic operculum and superior temporal gyrus overlaid on a T1-weighted MRI anatomical image in the Montreal Neurological Institute template.

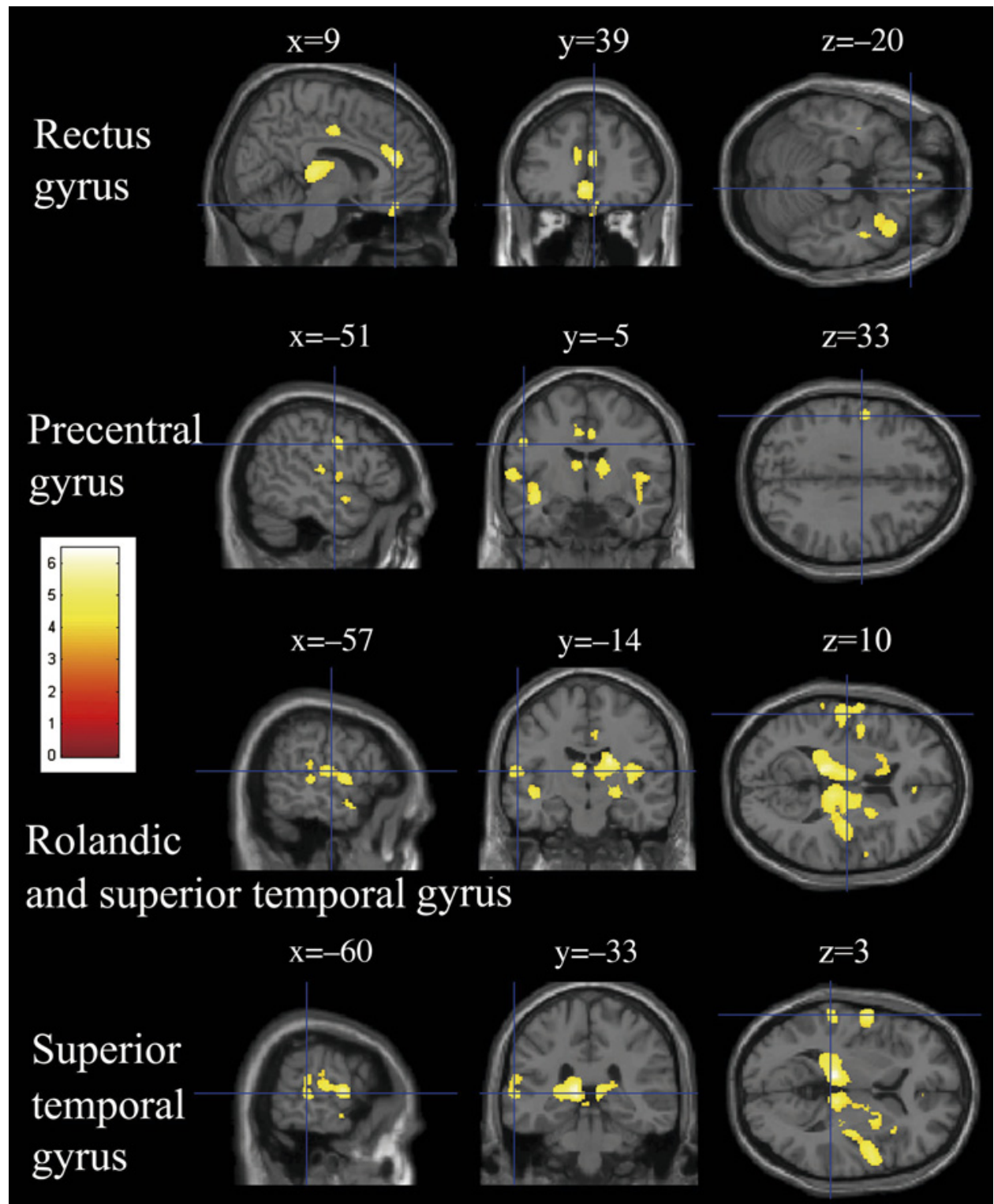

30-45 days of in-hospital rehabilitation following an acute exacerbation of COPD. At the time of data collection, patients were in stable condition. Among these patients, 12 discharged patients were recruited during their rest at home and 13 patients were recruited when they were awaiting discharge from hospital. Patients were diagnosed in Zhongshan Hospital (Xiamen, China) according to the diagnostic criteria of Global Initiative for Chronic Obstructive Lung Disease. ${ }^{14}$ Twenty-five healthy volunteers, with comparable age, gender and educational background, comprised the control group. All the subjects were free from a known history of cerebrovascular accident, heart failure, neurological disorders, obstructive sleep apnoea, coronary artery disease, diabetes or other diseases known to affect cognition. Patients were provided with therapy including inhaled ipratropium bromide, bricanyl, ventoline and budesonide. Demographic characteristics of the patients and healthy volunteers were listed in table 1 . Procedures were fully explained, and all subjects were provided written informed consent before participating in the study. The experimental protocol was approved by the Research Ethics Review Board of Xiamen University.

\section{Physiological and neuropsychological tests}

Physiological and neuropsychological tests and activities of daily living (ADL) (score range 14-56) ${ }^{15}$ were conducted 1 day before the MRI scan. Physiological tests include pulse rate and arterial blood pressure measures, arterial blood gas analysis and pulmonary function measure. Blood samples were taken in the morning between 07:00 and 07:30 h. The neuropsychological tests include (1) the Chinese version of the Mini-Mental State Examination (MMSE) that measured the general cognitive function and (2) the visual reproduction test, figure memory test and digital span forward and backward tasks, which, taken from the Chinese revised version of the Wechsler Memory Scale, were used to measure visual construction ability, visuospatial memory and short-term working memory, respectively. ${ }^{16}$ All data 
Figure 3 Grey matter density decrease in patients with chronic obstructive pulmonary disease versus healthy controls. Threedimensional slices depicting regions showing reduced grey matter in the bilateral anterior and middle cingulate gyri overlaid on a T1-weighted MRI anatomical image in the Montreal Neurological Institute template.

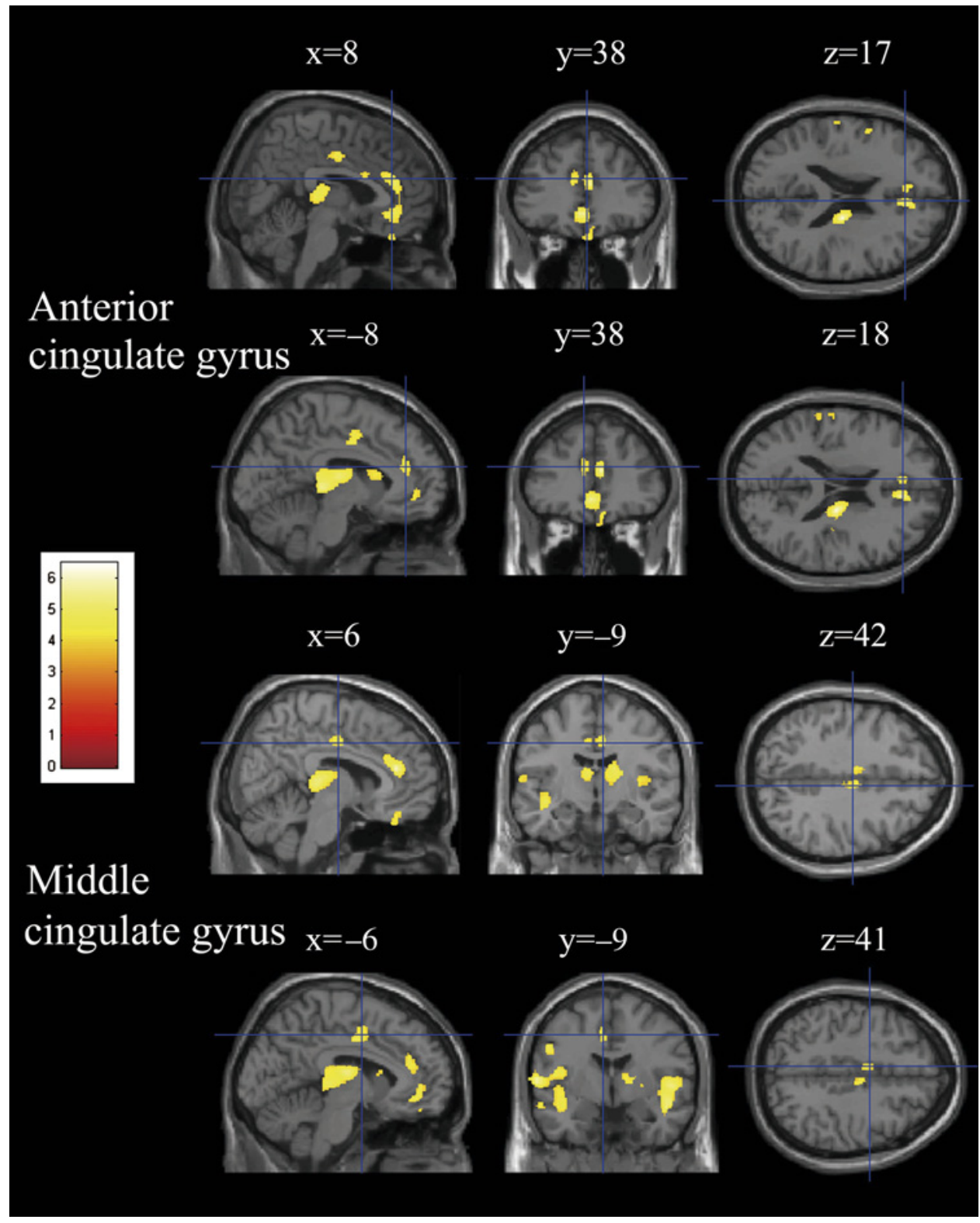

were analysed using SPSS V.19.0. Independent t test measured between-group differences. Statistical significance was set at $\mathrm{p}<0.05$.

\section{MRI data acquisition}

Images were acquired on a Siemens Trio Tim 3.0T (Erlangen, Germany) at MRI Research Center (Zhongshan Hospital). A three-dimensional (3D) structural MRI was acquired from each subject using a T1weighted MPRAGE sequence (TR/TE $=1900 \mathrm{~ms} /$ $2.48 \mathrm{~ms}, \mathrm{FOV}=25 \times 25 \mathrm{~cm}^{2}, \mathrm{NEX}=1$, matrix $=512 \times 256$, slice thickness $=1.0 \mathrm{~mm}$ ). Conventional two-dimensional $\mathrm{T} 1$ and T2 images were also acquired and examined for any incidental findings. A DTI pulse sequence with single shot diffusion-weighted echo planar imaging $\left(\mathrm{TR} / \mathrm{TE}=3600 / 95 \mathrm{~ms}, \quad \mathrm{FOV}=24 \times 24 \mathrm{~cm}^{2}, \quad \mathrm{NEX}=2\right.$, matrix $=128 \times 128$, slice thickness $=4.5 \mathrm{~mm}$ ) was applied sequentially in 30 non-collinear directions (b-value $=1000 \mathrm{~s} / \mathrm{mm}^{2}$ ) with one scan without diffusion weighting $\left(b=0 \mathrm{~s} / \mathrm{mm}^{2}\right)$. The following data analyses were conducted by two researchers who were blinded to the status of subjects.

\section{VBM analysis of 3D T1 images}

The 3D T1 images were used for GM analysis using VBM8 toolbox implemented in SPM8 (Wellcome Department of Imaging Neuroscience, University College London, London, UK). The following processing steps were carried out: (1) the images were inspected and set at the anterior commissure. Each reorientated image was segmented into GM, WM and cerebrospinal fluid in native space, and procrustes aligned GM images were generated by a rigid transformation. (2) The DARTEL registration method was used to create a study-specific template by using the aligned images from all the patients and controls to improve intersubject registration of structural images. ${ }^{9}$ The procedure implicated in six iterations, which began with the averaging of aligned data to generate an original template. Then, the first iteration of the registration 
Figure 4 Grey matter density decrease in patients with chronic obstructive pulmonary disease versus healthy controls. Threedimensional slices depicting regions showing reduced grey matter in the bilateral insula, bilateral thalamus and left caudate nucleus overlaid on a T1-weighted $\mathrm{MRI}$ anatomical image in the $\mathrm{MNI}$ template.

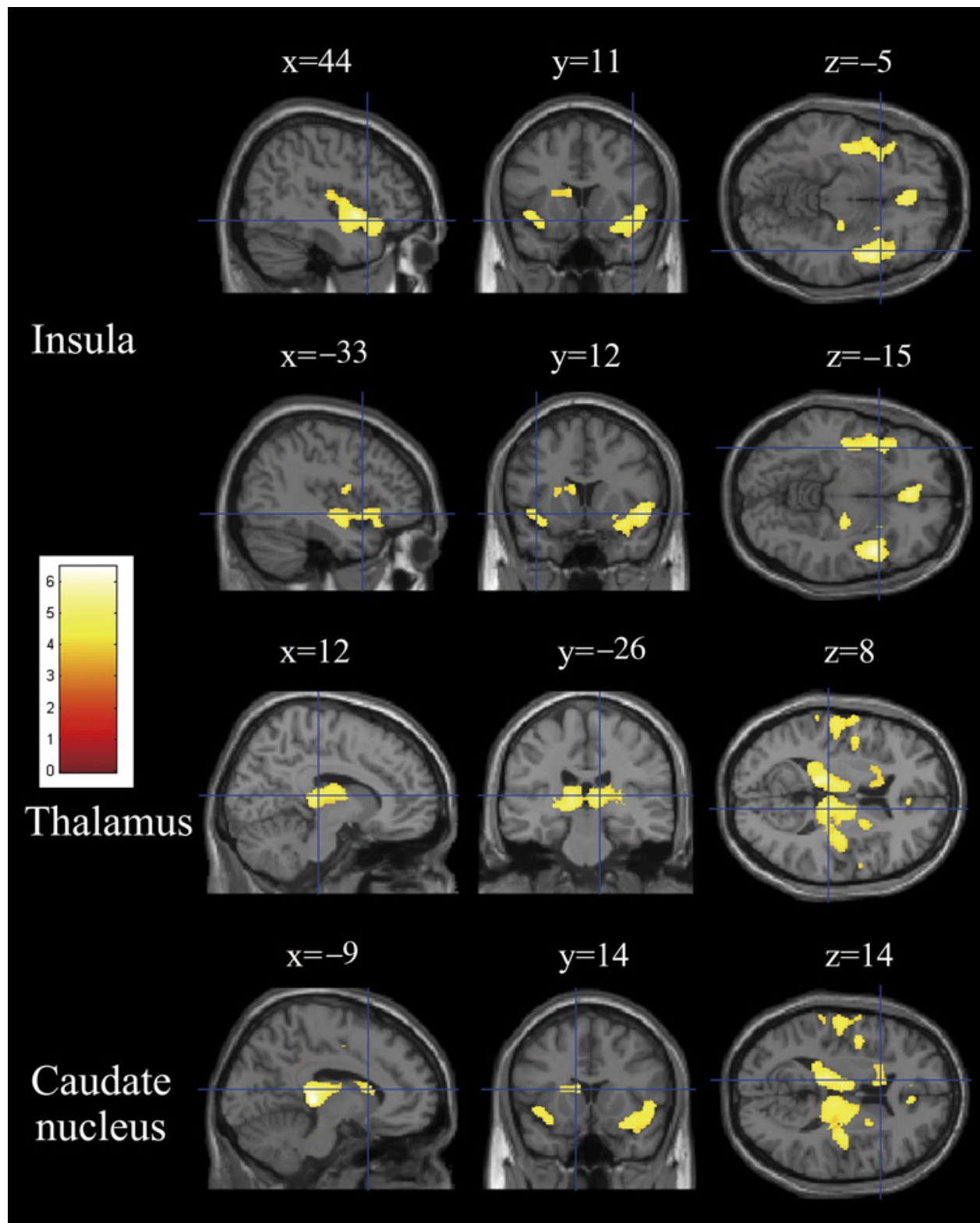

was completed on each subject and a new template was created. After this, the second iteration began. When six iterations were finished, the template was generated, which was the average of the DARTEL registered data. During iterations, all images were warped to the template yielding a series of flow fields that parameterised deformations. (3) The normalised images were transformed into Montreal Neurological Institute space. These GM images were then smoothed using a Gaussian kernel of $8 \mathrm{~mm}$ full-width at half-maximum. Independent $t$ tests were performed to examine between-group differences. The statistical parametric map was generated with the voxel level threshold at $\mathrm{t}>3.7734 \mathrm{p}<0.01$ (false discovery rate FDR correction with gender, age, education and total intracranial volume as covariates).

To analysed the correlation of GM image values with cognitive or physiological measurement, the following steps were first taken: (1) regions-of-interests were created for clusters showing differences between groups and (2) using these regions-of-interests masks, the GM values were extracted from each individual's normalised and smoothed GM maps. Then, the correlations were analysed using SPSS. Statistical significance was set at $\mathrm{p}<0.05$, with gender, age and education as covariates.

\section{TBSS analysis of DTI}

DCM2MII was used to convert diffusion tensor images from the proprietary scanner format to the NIFTI format. Then, the images were processed using the FSL 4.1.5 software package (http://www.fmrib.ox.ac.uk/fsl/). The images were realigned to the b-value (b0) image by affine transformations using FMRIB's diffusion toolbox ${ }^{17}$ to minimise distortions and reduce head motion artefacts. In order to remove non-brain tissue components and background noise, a brain mask was created from the first b0 image and then applied in the DTI to extract brain voxels using Brain Extraction Tool. After these processes, using DTIFit within the FMRIB's diffusion toolbox, the images were calculated to get the FA, $\lambda 1$ (longitudinal 
Table 3 Regional information of decreased grey matter density (cluster size $>100$ voxels) in patients with COPD compared with healthy controls

\begin{tabular}{|c|c|c|c|c|c|c|}
\hline \multirow[b]{2}{*}{ Area } & \multirow[b]{2}{*}{ Volume $\left(\mathrm{mm}^{3}\right)$} & \multirow[b]{2}{*}{ Brodmann areas } & \multicolumn{3}{|c|}{ MNI coordinate } & \multirow[b]{2}{*}{ t-score (peak) } \\
\hline & & & $x$ & $\mathbf{y}$ & $\mathbf{z}$ & \\
\hline Rectus_R & 118 & 11 & 9 & 39 & -20 & 4.31 \\
\hline Precentral_L & 100 & 6 & -51 & -5 & 33 & 4.95 \\
\hline Cingulum_Ant_R & 485 & 32 & 8 & 38 & 17 & 5.88 \\
\hline Cingulum_Ant_L & 212 & 32 & -8 & 38 & 18 & 4.49 \\
\hline Cingulum_Mid_R & 157 & 24 & 6 & -9 & 42 & 4.87 \\
\hline Cingulum_Mid_L & 136 & 24 & -6 & -9 & 41 & 4.91 \\
\hline Temporal_Sup_L & 280 & $22 / 42$ & -60 & -33 & 3 & 4.99 \\
\hline $\begin{array}{l}\text { Temporal_Sup/ } \\
\text { Rolandic_Oper_L }\end{array}$ & 837 & 22 & -57 & -14 & 10 & 4.91 \\
\hline $\begin{array}{l}\text { Insula/Temporal_Sup/ } \\
\text { Rolandic_Oper_R }\end{array}$ & 2821 & $13 / 22 / 47$ & 44 & 11 & -5 & 5.15 \\
\hline $\begin{array}{l}\text { Insula/Temporal_Sup/ } \\
\text { Rolandic_Oper_L }\end{array}$ & 1551 & $13 / 22 / 47$ & -33 & 12 & -15 & 4.48 \\
\hline Thalamus/pulvinar_L & 1270 & & -12 & -30 & 3 & 6.46 \\
\hline Thalamus/pulvinar_R & 2210 & & 12 & -26 & 8 & 5.29 \\
\hline Caudate_L & 212 & & -9 & 14 & 14 & 4.19 \\
\hline
\end{tabular}

diffusivity) (the magnitude of diffusion along the principal diffusion direction) and $\lambda 23$ (radial diffusivity) (the magnitude of diffusion in the two orthogonal directions perpendicular to the principal diffusion direction) maps. The whole-brain voxel-wise statistic analysis of the FA images was performed using TBSS in FSL. ${ }^{8}$ TBSS processing includes the following steps: (1) align the FA images of all subjects to a template that was arbitrarily selected from those FA images by non-linear registrations; (2) transform all the aligned FA images into $1 \times 1 \times 1 \mathrm{~mm}^{3}$ MNI152 space by affine registrations to remove the effect of cross-subject spatial variability that remains after the non-linear registration; (3) create the mean FA image and filter to retain only the centre of the WM tracts, with the threshold $\mathrm{FA} \geq 0.20$, and successfully exclude voxels, which consisted of GM or cerebrospinal fluid in the majority of subjects, so as to create the mean FA skeleton $^{8}$; (4) project individual subjects' FAs onto mean FA skeleton; (5) following these steps, data were fed into voxel-wise cross-subject statistical analyses. In all cases, the null distribution was built up over 5000 permutations, with significance analysed using independent $\mathrm{t}$ tests at $\mathrm{p}<0.05$ levels, uncorrected for multiple comparisons. We determined the anatomic localisation of each cluster by means of the FSL atlas tool, which incorporates several anatomic templates, including the Harvard-Oxford Cortical Structural Atlas, Harvard-Oxford Subcortical Structural Atlas, Talairach Daemon Labels and MNI Structural Atlas.

Within the cluster of changed FA, mean $\lambda 1$ and $\lambda 23$ values were extracted from each individual's $\lambda 1$ and $\lambda 23$ maps. Values were analysed using SPSS. Analysis of variance statistic was used to identify the group differences for these distinct brain locations. Statistical significance was set at $\mathrm{p}<0.05$.

\section{RESULTS}

\section{Physiological and behavioural findings}

Compared with the controls, independent t test showed that patients with COPD had significant decreases in arterial blood $\mathrm{SaO}_{2}$ and $\mathrm{Po}_{2}$, and increases in arterial blood $\mathrm{PCO}_{2}$ and heart rate. Patients with COPD had significantly lower values in one second over forced vital capacity (FVC), forced expiratory volume (FEV) and $\mathrm{FEV}_{1} / \mathrm{FVC}$ values and higher respiratory rate. The disease staging categories of patients with COPD based on $\mathrm{FEV}_{1} \%$ predicted were as follows: $\mathrm{FEV}_{1}=82 \%$ predicted, $\quad \mathrm{n}=1 ; \quad 54.9 \% \leq \mathrm{FEV}_{1}<78 \% \quad(64.9 \pm 7.6)$ predicted, $\mathrm{n}=8 ; \quad 31.9 \% \leq \mathrm{FEV}_{1}<48.4 \% \quad(42.0 \pm 5.4)$ predicted, $\mathrm{n}=7 ; \quad \mathrm{FEV}_{1}<29.9 \% \quad(26.2 \pm 2.9)$ predicted, $\mathrm{n}=9$. Patients with COPD had significantly lower scores in ADL, MMSE test, visual reproduction and figure memory (table 2).

\section{GM density}

No subject from either group showed visible abnormalities on T1-weighted structural images. VBM analysis showed that patients with COPD had decreased GM densities compared with healthy controls in the right gyrus rectus, left precentral gyrus, bilateral anterior and middle cingulate gyri, bilateral superior temporal gyri, bilateral anterior insula extending to Rolandic operculum (base of the pre- and post-central gyri), bilateral thalamus/pulvinars and left caudate nucleus (cluster size $>100$ voxels) (figures $1-4$, table 3 ).

\section{FA, longitudinal diffusivity and radial diffusivity in relation to COPD}

Whole-brain voxel-wise statistic analysis showed that patients with COPD had significantly lower FA in a broad range of brain regions compared with controls (figure 5, 
Figure 5 Statistical maps of group comparison of fractional anisotropy (FA) value on a voxelwise basis (results of Tract-Based Spatial Statistics). The group's mean FA skeleton (green) was overlaid on the Montreal Neurological Institute template. The threshold of mean FA skeleton was set at 0.2. Patients with chronic obstructive pulmonary disease show significantly lower FA value than healthy controls $(p<0.05)$.
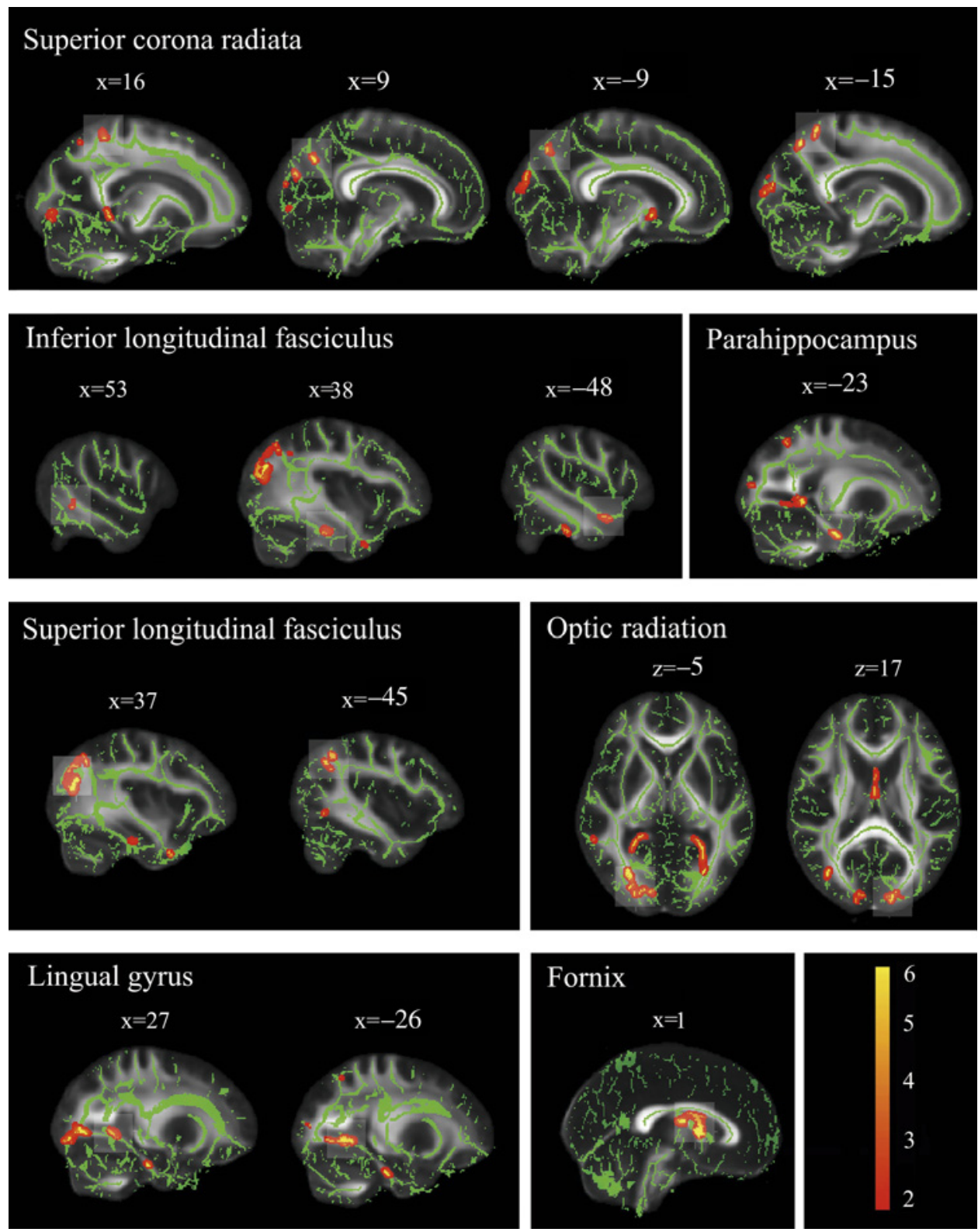

table 4). The significantly affected regions (clusters size $>40$ voxels) included the superior corona radiata (corresponding to bilateral precuneus and bilateral superior parietal lobules), superior longitudinal fasciculus (bilateral supramarginal gyri), inferior longitudinal fasciculus (left superior temporal gyrus, right middle temporal gyrus and fusiform gyrus), bilateral optic radiation, bilateral lingual gyri, left parahippocampal gyrus and fornix.

Lower FA values in these regions were associated with increased radial diffusivity and no changes of longitudinal diffusivity in patients with COPD versus controls (table 4).

\section{Correlations between MRI measurement and disease severity}

The correlations were listed in table 5. In patients with COPD, partial correlation (controlling for disease dura- tion, $\mathrm{FEV}_{1} / \mathrm{FVC}$, age, education and gender) revealed that the GM density in the bilateral anterior cingulate cortex, left superior temporal cortex, bilateral insula/superior temporal/Rolandic operculum, bilateral thalamus/ pulvinar and left caudate nucleus had positive correlations with arterial blood $\mathrm{Po}_{2}$. Partial correlation (controlling for $\mathrm{Po}_{2}, \mathrm{FEV}_{1} / \mathrm{FVC}$, age, education and gender) revealed that the GM density in the bilateral anterior cingulate cortex, right insula/superior temporal/Rolandic operculum and right thalamus/pulvinar had negative correlations with disease duration. Partial correlation (controlling for age, education and gender) analysis showed that the GM density in the left superior temporal lobes and left insula/ superior temporal/Rolandic operculum in patients with COPD was significantly correlated with figure memory score and the GM density in left precentral gyrus and left thalamus/pulvinar in patients with COPD correlated significantly with visual reproduction. 


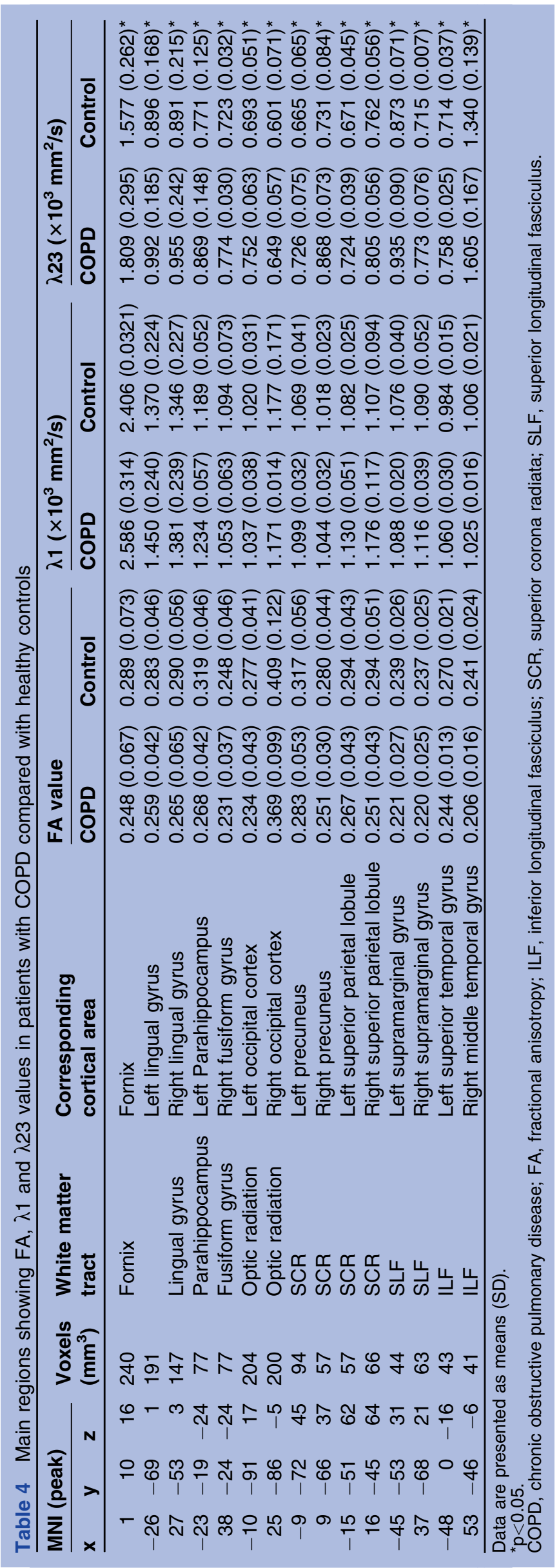

\section{DISCUSSION}

Our present study revealed that patients with COPD had decreased regional GM density confined to the limbic and paralimbic structures. GM density in impaired regions in patients with COPD had significant positive correlation with arterial blood $\mathrm{Po}_{2}$ and negative correlation with disease duration. The decreased WM FA value with increased radial diffusivity value was detected mainly in the visual cortex of the occipital lobe, the posterior parietal lobe as well as the temporal lobe. Decreased FA was associated with compromised myelin structure, changes in axonal morphologic structure and altered interaxonal spacing of fibre bundles. ${ }^{18}$ Radial diffusivity measures motion of water molecules perpendicular to fibres, and an increase of radial diffusivity is interpreted as abnormalities in myelinated membranes. ${ }^{19}$ Consequently, decreased FA and increased radial diffusivity in COPD indicated the impairment of WM microstructural integrity.

Previously, Borson et $a l^{6}$ only measured the volume of hippocampus in patients with COPD using region-ofinterest analysis and did not find any significant change. The impaired brain regions in COPD have also been found in other chronic hypoxic diseases. For example, decrease in GM volume/concentration in the gyrus rectus, precentral gyrus, anterior cingulate cortex, multiple sites within the temporal lobes, insular cortex, thalamus and caudate nucleus were detected in patients with obstructive sleep apnoea. ${ }^{13}$ Impairments of WM microstructure in the temporal lobe, parietal lobe, fornix and corona radiata were found in patients with congenital central hypoventilation syndrome. ${ }^{12}$ In our previous study, the decrease in GM volume in the anterior insula, anterior cingulate cortex and precentral cortex were found in high-altitude residents. ${ }^{10}$

GM density in impaired regions in patients with COPD had a strongly positive correlation with the arterial blood $\mathrm{Po}_{2}$, which suggested that the impairment in GM may result from low blood oxygen. Moreover, the GM density in some impaired regions showed negative correlations with disease duration. It is already known that hypoxia can induce metabolic decreases ${ }^{3-5}$ and cerebral perfusion decline ${ }^{2}$ in COPD. In addition, patients with COPD often suffer from systemic inflammation, which can exacerbate neuronal injury. ${ }^{1}$ A greater proportion of regions showing GM loss located in limbic/paralimbic cortex in patients with COPD may be due to the fact that phylogenetically older regions of the brain showed sharper vascular responses to hypoxia than evolutionary younger regions. ${ }^{20}$

A larger cortical network including the anterior insula and anterior cingulate cortex underlie the perception of dyspnoea, ${ }^{11}$ and these regions play an important role in regulating the cardiovascular system. ${ }^{21}$ Posterior thalamus was implicated in suppressing the ventilatory response to hypoxia. ${ }^{22}$ Hippocampus has been proved to control arterial pressure and heart rate. ${ }^{23}$ Thus, the morphological impairments in these regions may play 
Table 5 Correlations of grey matter density in impaired regions with $\mathrm{Po}_{2}$, disease duration and cognitive performances in patients with COPD

\begin{tabular}{|c|c|c|c|c|}
\hline \multirow[b]{2}{*}{ Area } & \multirow[b]{2}{*}{$\mathrm{Po}_{2}$ (patients) } & \multirow{2}{*}{$\begin{array}{l}\text { Disease duration } \\
\text { (patients) }\end{array}$} & \multicolumn{2}{|c|}{ Cognitive performances (patients + controls) } \\
\hline & & & Figure memory & Visual reproduction \\
\hline Precentral_L & & & & $r=0.306, p=0.028$ \\
\hline 2Cingulum_Ant_R & $r=0.530, p=0.021$ & $r=-0.471, p=0.038$ & & \\
\hline 1Cingulum_Ant_L & $r=0.744, p=0.001$ & $r=-0.476, p=0.036$ & & \\
\hline Cingulum_Mid_R & & & & \\
\hline Cingulum_Mid_L & & & & \\
\hline 11Temporal_Sup_L & $r=0.713, p=0.001$ & & $\mathrm{r}=0.511, \mathrm{p}=0.001$ & \\
\hline Temporal_Sup/ & & & & \\
\hline Rolandic_Oper_L & & & & \\
\hline 6Insula/Temporal_Sup/ & $r=0.615, p=0.007$ & & & \\
\hline Rolandic_Oper_R & & & & \\
\hline $\begin{array}{l}\text { 7Insula/Temporal_Sup/ } \\
\text { Rolandic_Oper_L }\end{array}$ & $r=0.656, p=0.004$ & & $r=0.498, p=0.001$ & \\
\hline 9Thalamus/pulvinar_L & $r=0.487, p=0.033$ & $r=-0.474, p=0.037$ & & $r=0.284, p=0.044$ \\
\hline 8Thalamus/pulvinar_R & $r=0.502, p=0.028$ & $r=-0.517, p=0.024$ & & \\
\hline 5Caudate_L & $r=0.550, p=0.017$ & & & \\
\hline
\end{tabular}

a role in respiratory and cardiovascular disturbances, such as higher heart rate and higher respiratory rate, in patients with COPD tested in our study.

In the present study, patients with COPD had poorer performance in MMSE, visuospatial memory and visual construction task. These results were consistent with those found in previous studies in patients with COPD. ${ }^{7}$ In line with the present findings of COPD, we previously found that long-time living in mild high-altitude hypoxic environment only impaired cognitive performances confined to visual reproduction and short-time complex figure memory. ${ }^{24}$ In our present study, the decreases in GM density in the frontal precentral cortex, insula/superior temporal cortex/Rolandic operculum and thalamus/ pulvinar may be responsible for the performance deficit in visual-related tasks since the GM density in these areas showed a significant positive correlation with figure memory or visual reproduction score. The following data support our findings: (1) recent research has identified the inferior frontal cortex served as a source of top-down modulation underlying attention to visual features ${ }^{25}$; (2) studies on patients using fMRI and positron emission tomography demonstrated Rolandic operculum as one of the visual structures ${ }^{26}$; (3) the pulvinar region of the thalamus is known to project to posterior parietal lobe and inferior temporal lobe. The pulvinar has been implicated in various visual functions in lesion studies. ${ }^{27}$ Declines in memory and executive function make contributions to declines in ADL. ${ }^{28}$ Visual construction tasks reflect executive function. Therefore, the decreases in GM density in the above regions that relate to visual construction may also be responsible for ADL deficits.

Our present study found the impairments of WM limited to the pathways of visual processing, including optic radiation, posterior parietal lobe (superior parietal lobule, supramarginal gyrus and precuneus) and the inferior temporal fusiform and lingual gyri. Visual information enters the primary visual cortex via optic radiation to the visual cortex. Cortical areas along the posterior parietal 'dorsal stream' are primarily concerned with spatial localisation and directing attention, while cortical areas along the inferior temporal 'ventral stream' are mainly concerned with the recognition and identification of visual stimuli. ${ }^{29}$ COPD also showed impaired WM in the middle temporal gyrus. Middle temporal cortex is important for the long-term build-up of perceptual memory for ambiguous motion stimuli. ${ }^{30}$ Based on the above data, our findings in WM may also clarify the mechanisms underlying the deficit in visual-related tasks. In addition, impaired WM in input and output fibres of hippocampus (fornix) may be related to the deficit in MMSE. Previous study on patients with Alzheimer's disease found that the volumes of hippocampus were significantly reduced and the volumes of the left hippocampus correlated significantly with the MMSE score. ${ }^{31}$ The limitation of our study is the weak statistical power of FA value analysis because the results obtained in the TBSS analysis could not survive multiple comparison correction.

\section{CONCLUSIONS}

In summary, we first demonstrated that COPD extended beyond the lung to the brain, with the decrease of regional GM density accompanied by impairment in the WM microstructural integrity. Our findings suggest significant participation of these structures in responding to hypoxic challenges, which include cardiovascular and air-hunger components. The brain structural changes may also underlie the psychological and mood changes of COPD.

\section{Author affiliations}

${ }^{1}$ Department of Physiology, Medical College of Xiamen University, Xiamen, China 
${ }^{2}$ Department of Neurology, the Affiliated Hospital of Hangzhou Normal University, Hangzhou, China

${ }^{3}$ Magnetic Resonance Center, Zhongshan Hospital, Medical College of Xiamen University, Xiamen, China

${ }^{4}$ Department of Respiratory, Zhongshan Hospital, Medical College of Xiamen University, Xiamen, China

${ }^{5}$ Department of Brain Protection and Plasticity, Institute of Basic Medical Sciences, Beijing, China

Contributors $\mathrm{HZ}$ contributed to conception and design, the accuracy of the data analysis, drafting the article and final approval of the version to be published. XW contributed to the study design, revising the article critically for important intellectual content and final approval of the version to be published. $\mathrm{JL}, \mathrm{YS}, \mathrm{YH}, \mathrm{TY}, \mathrm{SZ}$ and MF contributed to acquisition of data, revising the article critically for important intellectual content and final approval of the version to be published. JZ contributions to conception and design, acquisition of data and interpretation of data; drafting the article and revising it critically for important intellectual content; and final approval of the version to be published.

Funding This work was supported by National Science Foundation of China (Project No. 31071041; 81171324), Bureau of Science and Technology of Hangzhou (20090833B10) and Health Bureau of Zhejiang Province (2009A168).

Competing interests None.

Patient consent Obtained.

Ethics approval The experimental protocol was approved by the Research Ethics Review Board of Xiamen University.

Provenance and peer review Not commissioned; externally peer reviewed.

Data sharing statement We are pleased to share our data with other researchers. Jiaxing Zhang et al.

\section{REFERENCES}

1. Barnes PJ. Chronic obstructive pulmonary disease: effects beyond the lungs. PLoS Med 2010;7:e1000220.

2. Ortapamuk $\mathrm{H}$, Naldoken $\mathrm{S}$. Brain perfusion abnormalities in chronic obstructive pulmonary disease: comparison with cognitive impairment. Ann Nucl Med 2006;20:99-106.

3. Shim TS, Lee JH, Kim SY, et al. Cerebral metabolic abnormalities in COPD patients detected by localized proton magnetic resonance spectroscopy. Chest 2001;120:1506-13.

4. Sicard KM, Duong TQ. Effects of hypoxia, hyperoxia, and hypercapnia on baseline and stimulus-evoked BOLD, CBF, and CMRO2 in spontaneously breathing animals. Neuroimage 2005;25:850-8.

5. Sinha S, Kumar V, Jagannathan NR, et al. Proton magnetic resonance spectroscopy of brain to study the cerebral metabolic abnormalities in COPD patients: a case control study in north India. Indian J Chest Dis Allied Sci 2009;51:15-19.

6. Borson S, Scanlan JM, Friedman S, et al. Modeling the impact of COPD on the brain. Int J Chron Obstruct Pulmon Dis 2008;3:429-34.

7. Dodd JW, Getov SV, Jones PW. Cognitive function in COPD. Eur Respir J 2010;35:913-22.

8. Smith SM, Jenkinson M, Johansen-Berg $\mathrm{H}$, et al. Tract-based spatial statistics: voxelwise analysis of multi-subject diffusion data. Neuroimage 2006;31:1487-505.

9. Ashburner J. A fast diffeomorphic image registration algorithm. Neuroimage 2007;38:95-113.
10. Zhang J, Yan X, Shi J, et al. Structural modifications of the brain in acclimatization to high-altitude. PLoS One 2010;5:e11449.

11. von Leupoldt A, Dahme B. Cortical substrates for the perception of dyspnea. Chest 2005;128:345-54.

12. Kumar R, Macey PM, Woo MA, et al. Diffusion tensor imaging demonstrates brainstem and cerebellar abnormalities in congenital central hypoventilation syndrome. Pediatr Res 2008;64:275-80.

13. Joo EY, Tae WS, Lee MJ, et al. Reduced brain gray matter concentration in patients with obstructive sleep apnea syndrome. Sleep 2010;33:235-41.

14. Pauwels RA, Buist AS, Calverley PM, et al. Global strategy for the diagnosis, management, and prevention of chronic obstructive pulmonary disease. NHLBI/WHO Global Initiative for Chronic Obstructive Lung Disease (GOLD) Workshop summary. Am J Respir Crit Care Med 2001;163:1256-76.

15. Lawton MP, Brody EM. Assessment of older people: self-maintaining and instrumental activities of daily living. Gerontologist 1969;9:179-86.

16. Gong YX. Manual for the Wechsler Memory Scale-Revised. Changsha, Hunan, China: Hunan Medical University, 1989.

17. Jenkinson $\mathrm{M}$, Smith $\mathrm{S}$. A global optimisation method for robust affine registration of brain images. Med Image Anal 2001;5:143-56.

18. Beaulieu $C$. The basis of anisotropic water diffusion in the nervous system-a technical review. NMR Biomed 2002;15:435-55.

19. Song SK, Sun SW, Ramsbottom MJ, et al. Dysmyelination revealed through MRI as increased radial (but unchanged axial) diffusion of water. Neuroimage 2002;17:1429-36.

20. Binks AP, Cunningham VJ, Adams L, et al. Gray matter blood flow change is unevenly distributed during moderate isocapnic hypoxia in humans. J Appl Physiol 2008;104:212-17.

21. Nagai M, Hoshide S, Kario K. The insular cortex and cardiovascular system: a new insight into the brain-heart axis. J Am Soc Hypertens 2010;4:174-82.

22. Teppema LJ, Dahan A. The ventilatory response to hypoxia in mammals: mechanisms, measurement, and analysis. Physiol Rev 2010;90:675-754.

23. Macefield VG, Gandevia SC, Henderson LA. Neural sites involved in the sustained increase in muscle sympathetic nerve activity induced by inspiratory capacity apnea: a fMRI study. J Appl Physiol 2006;100:266-73.

24. Zhang J, Liu H, Yan X, et al. Minimal effects on human memory following long-term living at moderate altitude. High Alt Med Biol 2011;12:37-43.

25. Zanto TP, Rubens MT, Bollinger J, et al. Top-down modulation of visual feature processing: the role of the inferior frontal junction. Neuroimage 2010;53:736-45.

26. Fried I, Nenov VI, Ojemann SG, et al. Functional MR and PET imaging of rolandic and visual cortices for neurosurgical planning. $J$ Neurosurg 1995;83:854-61.

27. Arend I, Machado L, Ward R, et al. The role of the human pulvinar in visual attention and action: evidence from temporal-order judgment, saccade decision, and antisaccade tasks. Prog Brain Res 2008;171:475-83.

28. Tomaszewski Farias S, Cahn-Weiner DA, Harvey DJ, et al. Longitudinal changes in memory and executive functioning are associated with longitudinal change in instrumental activities of daily living in older adults. Clin Neuropsychol 2009;23:446-61.

29. Itti L, Koch C. Computational modelling of visual attention. Nat Rev Neurosci 2001;2:194-203.

30. Brascamp JW, Kanai R, Walsh V et al. Human middle tempora cortex, perceptual bias, and perceptual memory for ambiguous threedimensional motion. J Neurosci 2010;30:760-6.

31. Laakso MP, Soininen H, Partanen K, et al. Volumes of hippocampus, amygdala and frontal lobes in the MRI-based diagnosis of early Alzheimer's disease: correlation with memory functions. J Neural Transm Park Dis Dement Sect 1995;9:73-86. 